Wasserstoff, Stickstoff, Kohlensäure, Ammoniak; der Temperalurbereich $\operatorname{lag} z$ wischen $-79^{\circ}$ und $+151,5^{\circ}$.

Es ergab sich, daB für Wasserstoff 2 wischen $+80^{\circ}$ und $-80^{\circ}$ das Hen ry 'sche Verteilungsgesetz und fïr die drei anderen Gase bei konstanter Temperatur die Beziehung gilt:

$$
a_{i}=u p_{i}
$$

Die adsorbierte Menge lät sich für ein groBes Temperatur- und Druckgebiet befriedigend wiedergeben durch die Gleichung:

$$
\lg a_{t}=\log a_{o}-(\zeta-\xi \lg p) t \text {, }
$$

worin $\xi$ und $\xi$ in einer einfachen Beziehung $z u$ den obigen zwei Konstanten $\alpha$ und $\frac{1}{n}$ stehen.

Abweichungen hiervon zeigen sich erst bei nahe an dem Verllassigungspunkt der drei Gase liegenden Temperaturen.

Die für dieselben drei Gase gemessenen Adsorptionswärmen ergeben gute Uebereinstimmung mit den Werten, welche nach der von $H$. F reundlich thermodynamisch abgeleiteten Formel

$$
q=\frac{n R T^{2}}{\lg e}(\zeta-\xi \lg p)
$$

aus dem Verlauf der Isothermen berechnet wurden.

Diese Arbeit, sowie die oben referierte von J. F. Ho m f re y dürften in ihrer Nebeneinanderstellung ein wesentliches Interesse verdienen.

\section{Hans Brehm.}

Findlay, A., und Shen, B., Einflub ron Kolloiden und feinen Suspensionen ant die Idss. lichkeit von Gasen in Wasger. (Journ. Chem. Soc 101, $1459-1468,1912$.)

Verfasser untersuchen die Löslichkeit von $\mathrm{CO}_{2}$ in Lösungen von Chlorammonium, Chlorkalium, Chlorbarium, Ferroammoniumsulfat, Rohrzucker und Chloralhydrat bei verschiedenen Drucken und den Einflub der Kolloide Pepton, Propepton und Hămoglobin aut dieselbe. Ferner auch die Löslichkeit von Wasserstoff in Wasser bei Gegenwart von Dextrin, Stärke Gelatine, Eisenhydroxyd und Silbersuspensionen.

Während, wie in einer fruiheren Arbeit (A. Find 1 a y und Creighton. Trans. 97, 536, 1910) gezeigt war, die Lőslichkeil von $\mathrm{CO}_{2}$ und salpetriger Säure in Wasser bei Gegenwart von Kolloiden nicht den Henry' schen Gesetz gehorcht, so ist dies wohl der Fall bei der Löslichkeit von $\mathrm{CO}_{2}$ in Lösungen der oben angegebenen Kristalloide. Bei allen diesen Losungen wat die Löslichkeit des $\mathrm{CO}_{2}$ geringer als im Wasser, und zwar un so geringer, je konzentrierter die Lossung war.

Bemetkenswert ist der außergewöhnlich schwache Einfluß von Chloralhydrat auf die Löslichkeit von $\mathrm{CO}_{2}$. Ebenso mag erwahnt werden, daB, wenn man die Loslichkeit nicht auf das ganze Volumen der Losung, sondern nur auf das in der Losung vorhandene Wasser bezicht, die Löslichkeit von $\mathrm{CO}_{2}$ in einer Lösung von Chloralhydrat groBer ist als in reinem Wasser. Dies ist auch noch der Fall, selbst wenn man annimmt, dab das im Chloralhydrat gebundene Wasser abgespaltet wird und nun wie reines Wasser sich verhalt. Beide Tatsachen stimmen mit den von Knop am Wasserstoff beobachteten uberein.

Schon in einer fritheren Arbeit von A. Findlay und Creighton (Trans. loc. cit., Biochem. Journ. loc. cit.) ist gezeigt worden, dab die Loslichkeit von
$\mathrm{CO}_{2}$ in Gegenwart von Kolloiden nur dann betrachtlich gröber scheint als in reinem Wasser, wenn man das Entstehen einer neuen chemischen Verbindung annehmen kann.

Die Löslichkeit von $\mathrm{CO}_{2}$ in Losungen von Pepton, Propepton und Hämoglobin ist gröBer als in Wasser. nimmt aber mit wachsendem Druck ab. Dieses Verhalten ist ohne Zweifel einer chemischen Bindung z.uzuschreiben.

Was die Löslichkeit von Wasserstoff bei Gegenwart von Kolloiden anbelangt, so gehorcht diese, möglicherweise mit Ausnahme der Gelatinelosung, dem Henry'schen Gesetz; doch waren die zu diesen Untersuchungen verwandten Apparate zu primitiv. Die Gegenwart von $\mathrm{Fe}(\mathrm{OH})_{8}$ oder fein verteiltem Silber verursacht keinen merklichen EinfluB auf die Loslichkeit von Wasserstoff. Bei den anderen untersuchten Stoffen (Dextrin, Stärke, Gelatine) nimmt die Löslichkeit mit wachsender Konzentration der Lösung $a b$. Schucht.

Ma r c, R., Ueber die Adsorption an Kristallen. V. Ueber die Kristallisation aus wasserigen L. sungen. (Zeitschr. f. physik. Chem. 75, 732, 1911.)

Bekanntlich hatte der Verfasser nachgewiesen, daß dem Kristallisationsproze $B$ eine Adsorption der Lösung durch die Kristalle vorausgehe, da Fremdstoffe, die von den Keimen stark adsorbiert werden, die Kristallisation stark behindern und beeinflussen konnen. Daß diese Adsorption nach der von $H$. Freundlich wiederholt geprüften Isotherme vor sich geht, beweist der Verfasser durch die Untersuchung der Aufnahme von Farbstoffen durch schwerlösliche, aber wohlkristallisierte Stoffe. Bei allen diesen Adsorptionen ist aber ein Maximum zu beobachten, welches konstant bleibt, auch wenn die Konzentration der lobsung weiter erhöht wird. Er nennt dieses Maximum die Sättigungsgrenze der Adsorption. Er versucht sich theoretisch von dem Vorhandensein dieser Sättigungsgrenze Rechenschaft zu geben. An amorphen und flïssigen Stoficn sind die Oberflächenkräte uberall qualitativ gleicher Art bei kristallisierten aber nicht. Es missen vielmehr die verschiedenen Kristallflachen verschiedene Adsorptionsgrobe besitzen. Daher kommt es, daB die Reihenfolge der Adsorption für verschiedene gelöste Stoffe an verschieden kristallisierten Stoffen nicht die gleiche ist, wie H. Freundlich an amorphen Stoffen gefunden hatte. Det Verfasser versuchte deshalb $z u$ prufen, ob an isomorphen Stoffen vielleicht die gleiche Reihenfolge zu erkennen sei. Die Schwierigkeit der Untersuchung lieb das in einigen Fällen, aber nicht immer deutlich hervortreten. A. Lottermoser.

Marc, R., Bemerkungen zu einer Arbeit von G. C. Schmidt) ,Ueber Adsorption von Lösungen". (Zeitschr. f. physik Chem. 76, 58-66, 1911.)

Der Verfasser versucht nachzuweisen, dab die von G. C. Sch $m$ i d t aufgestellte Formel $\ln \frac{S}{S-c}-B x=C c$, die der Tatsache Rechnung tragen soll, daß bei den Adsorptionen schlieBlich ein Maximum erreicht wird, von dem an vom Adsorbens nichts mehr aus der Losung trotz Konzentrationssteigerung aufgenommen wird, keine Vorzüge vor der Freundlich'schen Formel besitzt. Denn bis zur Sättigungsgrenze gibt die letztere mindestens genau so gut die Versuchs-

J) Ref. Koll.-Zeitschr. 11, 129 (1912). 